OPEN ACCESS

Edited by:

Paul Kelley,

Open University, UK

Reviewed by:

Dong-Hoon Lee,

Yonsei University, South Korea

Emmanuel Mellet,

French National Centre for Scientific

Research (CNRS), France

*Correspondence:

Lijuan Zhang

lj.zhang@siat.ac.cn

Received: 03 August 2016 Accepted: 29 November 2016 Published: 20 December 2016

Citation:

Jiang C, Yi L, Su S, Shi C, Long X,

Xie $G$ and Zhang $L$ (2016) Diurnal Variations in Neural Activity of Healthy

Human Brain Decoded with

Resting-State Blood Oxygen Level

Dependent $\mathrm{FMRI}$

Front. Hum. Neurosci. 10:634.

doi: 10.3389/fnhum.2016.00634

\section{Diurnal Variations in Neural Activity of Healthy Human Brain Decoded with Resting-State Blood Oxygen Level Dependent fMRI}

\author{
Chunxiang Jiang ${ }^{1}$, Li Yi ${ }^{2}$, Shi Su' ${ }^{1}$, Caiyun Shi' ${ }^{1}$, Xiaojing Long ${ }^{1}$, Guoxi Xie ${ }^{1}$ and \\ Lijuan Zhang ${ }^{1 *}$ \\ ${ }^{1}$ Paul C. Lauterbur Research Center for Biomedical Imaging, Shenzhen Institutes of Advanced Technology, Chinese \\ Academy of Sciences, Shenzhen, China, ${ }^{2}$ Department of Neurology, Peking University Shenzhen Hospital, Shenzhen, China
}

It remains an ongoing investigation about how the neural activity alters with the diurnal rhythms in human brain. Resting-state functional magnetic resonance imaging (RS-fMRI) reflects spontaneous activities and/or the endogenous neurophysiological process of the human brain. In the present study, we applied the ReHo (regional homogeneity) and ALFF (amplitude of low frequency fluctuation) based on RS-fMRI to explore the regional differences in the spontaneous cerebral activities throughout the entire brain between the morning and evening sessions within a 24-h time cycle. Wide spread brain areas were found to exhibit diurnal variations, which may be attributed to the internal molecular systems regulated by clock genes, and the environmental factors including light-dark cycle, daily activities and homeostatic sleep drive. Notably, the diurnal variation of default mode network (DMN) suggests that there is an adaptation or compensation response within the subregions of DMN, implying a balance or a decoupling of regulation between these regions.

Keywords: diurnal rhythm, fMRI, ReHo, ALFF, neural activity

\section{INTRODUCTION}

Circadian rhythm of sleep and wakefulness is a fundamental property of human physiology, which is important for sustaining essential body functions. It has been reported that the physiology of human brain is rhythmic genetically and systematically (Smale et al., 2008). The suprachiasmatic nuclei $(\mathrm{SCN})$, located in a small region of the hypothalamus, is considered to be the primary circadian clock in mammals (Hastings et al., 2003; Saper et al., 2005). SCN activity fluctuates on a daily basis (Hofman and Swaab, 1993). Understanding system properties intrinsic to the $\mathrm{SCN}$, as well as its regulation of peripheral oscillators throughout the brain and body will provide a fundamental contribution to our understanding of the neurophysiological basis of human circadian behavior.

The fluctuating activities of SCN and peripheral oscillators, in concert with the learning and experience over the course of a day may influence neurons activity and the way brain regions communicate with each other. Resting-state functional magnetic resonance imaging (RS-fMRI) technique can investigate brain activity by measuring the variance in the spontaneous fluctuations of the blood oxygen level dependent (BOLD) signal. Based on RS-fMRI, regional homogeneity (ReHo) and amplitude of low frequency fluctuation (ALFF) methods have been used to analyze 
spontaneous low-frequency $(<0.08 \mathrm{~Hz}$ ) blood oxygenation leveldependent fluctuations of the brain (Zang et al., 2004; Yang et al., 2007). ReHo measures the coherence of low frequency fluctuation within a given area, which can be used to assess the synchrony of neural activity based on the hypothesis that brain activities would more likely occur in clusters than in a single voxel. ALFF is associated with field potential activity of local brain regions (Logothetis et al., 2001) that could be employed to evaluate the intensity of intrinsic or spontaneous neuronal activity of the brain (Yu-Feng et al., 2007). Therefore, ReHo and ALFF can serve as sensitive markers indicating the alteration of brain function. For example, ReHo decreases in the posterior cingulate cortex/precuneus $(\mathrm{PCC} / \mathrm{PCu})$ in the $\mathrm{AD}$ patients $(\mathrm{He}$ et al., 2007), ALFF increases in the anterior portion of the dorsal anterior cingulate cortex in subthreshold depression patients ( $\mathrm{Li}$ et al., 2014).

A recent research investigated if the brain networks are stable during a 24-h period by acquiring RS-fMRI data from 12 young adults at 3 -h intervals over 24 consecutive hours. The authors found resting state sub-network dynamicity, especially the dynamic fluctuations of default mode network (DMN) connectivity and speculated that connectivity may have different spatiotemporal properties on the neural level (Park et al., 2012). Marek et al. (2010) also reported that the orienting and executive attention neuronal networks present time-of-day related variations with a stroop-like task performed five times a day. In our previous work, we have found a diurnal variation pattern of white matter microstructure which may function as the substrates of the phasic neural activities in correspondence to the environment adaptation in a light-dark cycle (Jiang et al., 2014). These analyses of the spontaneous neural activities in response to the diurnal rhythm may provide a new insight toward better understanding of the brain physiology and sleep medicine. Therefore, we aim to investigate the dynamic characteristic of neural activity by evaluating the intra-individual variability of ReHo and ALFF over one light-dark cycle.

\section{MATERIALS AND METHODS}

\section{Subjects}

This study was approved by the institutional review board of Shenzhen Institutes of Advanced Technology. Informed consent was obtained from each of the 16 healthy subjects ( 6 males, 10 females, 23-31 years, mean age $24.8 \pm 2.0$ years) prior to the MRI examinations. All the subjects were graduate students whose daily activities follow a campus routine in which the regular meal time, bedtime (11:00 pm $\pm 1 \mathrm{~h}$ ), and taking classes for the rest time were followed. No participant was excluded because of neurological or psychiatric illnesses, sleep disorder, drug, coffee, smoking, or alcohol abuse.

\section{MRI Data Acquisition}

All the experiments were performed on a 3T Siemens MRI scanner (Siemens Trio system, Erlangen, Germany) with a 12channel head coil in a scan room with temperature controlled between 23 to $24^{\circ} \mathrm{C}$. High resolution T1 weighted whole brain scan was acquired using MPRAGE sequence for anatomical reference with TR/TE/TI 1900/2.53/900 ms, flip angle $9^{\circ}$, field of view (FOV) $250 \mathrm{~mm}$, slice thickness $1 \mathrm{~mm}$, acquisition matrix $256 \times 256$. Resting state BOLD images were acquired axially with an echo-planar imaging (EPI) sequence with TR/TE 3000/30 ms, flip angle $90^{\circ}$; FOV $210 \mathrm{~mm}$, matrix $128 \times 128$, 30 slices, thickness $3 \mathrm{~mm}$, bandwidth $1395 \mathrm{~Hz} /$ pixel, 60 volumes. Subjects were instructed to keep their eyes closed, relax their minds and remain motionless as much as possible but were requested not to fall asleep during the MRI data acquisition. Integrated parallel acquisition technique (iPAT) with acceleration factor of 2 was used to reduce the acquisition time and image distortion from susceptibility artifacts.

For each subject the MRI data was acquired in the morning at $8: 30 \mathrm{am} \pm 0.5 \mathrm{~h}$ and repeated in the evening at 7:30 $\mathrm{pm} \pm 0.5 \mathrm{~h}$ during a 24 -h interval, about $0.5-1 \mathrm{~h}$ after meal before the MRI acquisition.

\section{Data Preprocessing}

Data preprocessing was carried out using Data Processing Assistant for Resting-State fMRI (DPARSF ${ }^{1}$ ). The first 10 volumes of each time series were discarded for the instability of the initial MRI signal and subjects' adaptation. The remained fMRI data were corrected for within-scan acquisition time differences between slices. After head motion corrected by realignment of all consecutive volumes to the first image [using a least square approach and a six parameter (rigid body) spatial transformation], the fMRI data were coregistered (rigid-body transformation) to T1-weighted images, and then spatially normalized (12-parameter affine transformation) to the MNI (Montreal Neurological Institute) space and resample to $3 \mathrm{~mm} \times 3 \mathrm{~mm} \times 3 \mathrm{~mm}$. Temporal band-pass filtering (0.01$0.08 \mathrm{~Hz}$ ) was then used to remove the linear trend of time courses to reduce low-frequency drift and physiological high frequency respiratory and cardiac noise. ReHo was defined by Kendall's coefficient of concordance (KCC) which was used to measure ReHo of the time series of a given voxel with those of its nearest neighbors according to formula 1 (Zang et al., 2004). In order to reduce the effect of individual variability, ReHo was divided by the global mean value for each subject. Then the ReHo map was smoothed with a Gaussian filter of $4 \mathrm{~mm}$ full width at half-maximum (FWHM).

$$
W=\frac{\sum R_{\mathrm{i}}^{2}-n(\bar{R})^{2}}{\frac{1}{12} K^{2}\left(n^{3}-n\right)}
$$

where $W$ is the KCC among given voxels, ranged from 0 to $1 ; R_{\mathrm{i}}$ is the sum rank of the $i$ th time point; where $\bar{R}=((n+1) K) / 2$ is the mean of the $R_{\mathrm{i}}$ 's; $K$ is the number of time series within a measured cluster (27, one given voxel plus the number of its neighbors); $n$ is the number of ranks (here $n=50$ ).

The filtered time series were converted to a frequency domain using a Fast Fourier Transform. Then the square root of power spectrum was calculated and averaged within the frequency range between 0.01 and $0.08 \mathrm{~Hz}$ at each voxel. ALFF was defined by the

\footnotetext{
${ }^{1}$ http://www.restfmri.net
} 
averaged square root. In order to reduce the effect of variability across participants, the ALFF of each voxel was divided by the global mean ALFF value. A more detailed methodology of ALFF can be found in previous studies (Yang et al., 2007; Yu-Feng et al., 2007).

\section{Statistical Analysis}

A one sample $t$-test was performed within each scan session to explore which brain regions show higher ReHo/ALFF than global mean value $(p<0.001$, FDR corrected). Paired $t$-test was applied to compare the ReHo/ALFF difference between the morning and evening scan sessions. Voxels with $p<0.05$ and cluster size $>85$ voxels were considered to be statistically significant (corrected by AlphaSim program). Two statistic parametric files were obtained during the paired $t$-test in ReHo and ALFF, which were then used to extract the overlapped brain regions in $\mathrm{ReHo}$ and ALFF by a homemade program implemented in MATLAB (Mathworks, Natick, MA, USA). Parametric data was mapped to the T1 weighted images to facilitate visualization.

\section{RESULTS}

\section{Diurnal Variations of ReHo}

The results of inner-group ReHo analysis were shown in Figure 1. Voxels in the middle occipital gyrus and the DMN including PCC, PCu, medial prefrontal cortex (MPFC) and bilateral inferior parietal lobe (IPL) exhibited significantly higher ReHo relative to the rest brain areas. The neural activity of the AM and PM sessions were comparable in pattern but different in strength.

Compared to the PM session, the AM data showed a significant ReHo increase in the areas including bilateral middle and inferior occipital gyrus (BA17, BA18, BA19), lingual gyrus, fusiform gyrus, precentral and postcentral gyrus, cuneus and paracentral lobule, left middle and superior temporal gyrus and decreased in bilateral superior and middle frontal gyrus, medialand inferior-orbital frontal gyrus, superior frontal gyrus, anterior cingulate, PCC, $\mathrm{PCu}$, parahippocampal gyrus, hippocampus, caudate, amygdala and right inferior-orbital frontal gyrus (Figure 2) (summarized in Tables 1 and 2). In summary, the ReHo mainly increased in the morning session in the occipital, parietal and left temporal lobes, and decreased in frontal and anterior-posterior cingulate midline.

\section{Diurnal Changes of ALFF}

For the inner-group ALFF analysis, significant increased ALFF was shown in PCC, MPFC, PCu, and the non-specific areas including ventricles and cisterns (Figures 3A,C) within AM and PM sessions. As ALFF is sensitive to physiological noise irrelevant to brain activity (Zou et al., 2008), fractional ALFF (fALFF) method, a ratio of the power of each frequency at the low-frequency range $(0.01-0.08 \mathrm{~Hz})$ to that of the entire frequency range, was applied to suppress the non-specific signal components in the rs-fMRI and improve the sensitivity in detecting regional spontaneous brain activity. Higher fALFF was found in bilateral occipital lobes, PCC, PCu, cuneus, IPL, MPFC, middle and superior temporal gyrus, precentral and postcentral gyrus, as shown in Figures 3B,D.

Compared to the PM session, significant increased morning ALFF occurred in left precentral and postcentral gyrus, middle and superior temporal gyrus, bilateral paracentral lobule, lingual gyrus, cuneus, superior and middle and inferior occipital gyrus, and decreased in the area including bilateral anterior cingulate, rectus, medial- and inferior-orbital frontal gyrus, superior frontal gyrus, and right posterior cingulate, amygdala, hippocampus, parahippocampal gyrus, $\mathrm{PCu}$, middle and inferior temporal gyrus (Figure 4) (summarized in Tables 3 and 4).

\section{Brain Areas with Both ReHo and ALFF Variations}

Compared to the PM session, ReHo and ALFF of the morning session were both increased in bilateral middle and inferior occipital gyrus (BA17, BA18, BA19), lingual gyrus, cuneus, paracentral lobule, left precentral and postcentral gyrus, left middle and superior temporal gyrus, and decreased in bilateral anterior cingulate, superior frontal gyrus, rectus, medial- and inferior-orbital frontal gyrus, amygdala, right PCC, inferior temporal gyrus and parahippocampal gyrus (Figure 5).

\section{DISCUSSION}

The current study demonstrated the diurnal rhythmic dynamic of the neural activity of human brain. Circadian rhythms are widespread in human brain and influenced by the interactions between internal molecular systems and environmental cues (Cirelli, 2009; Mohawk et al., 2012). Core clock genes are known to comprise transcriptional-translational auto-regulatory complexes. These genes make up a group of auto-regulatory loops with diurnal variation of activity and present rhythmic expression of their own and their regulatory target transcrips. Li et al. (2013) reported that there exists a rhythmic rise and fall in the transcriptional activity of hundreds of genes [including three Period homolog (PER 1-2-3) genes; brain and muscle Arntlike protein-1 (BMSL1) and so on] initiating or responding to the regulation of 24-h behavioral and hormonal cycles. Vicentic et al. (2005) also found that CART (cocaine- and amphetamineregulated transcript) peptides exhibit a diurnal rhythm in several brain regions including nucleus accumbens, hypothalamus and amygdala. Muto et al. (2016) investigated the local modulation of human brain responses by circadian rhythmicity and sleep debt. They found that the response of some subcortical regions like thalamus, head of caudate nucleus and putamen follows a $24 \mathrm{~h}$ circadian rhythmicity, and some brain regions were affected significantly by sleep debt, and some are affected by the interaction of the two factors (Muto et al., 2016). The circadian rhythmicity may substrate the physiological mechanisms of the diurnal variation of ReHo and amplitude fluctuation of neural activity occurred in some of the structures mentioned above in the current study.

The DMN is supposed to be involved in self-referential functions such as internal mentation, recollection and imagination and conceptual processing, containing a set of 


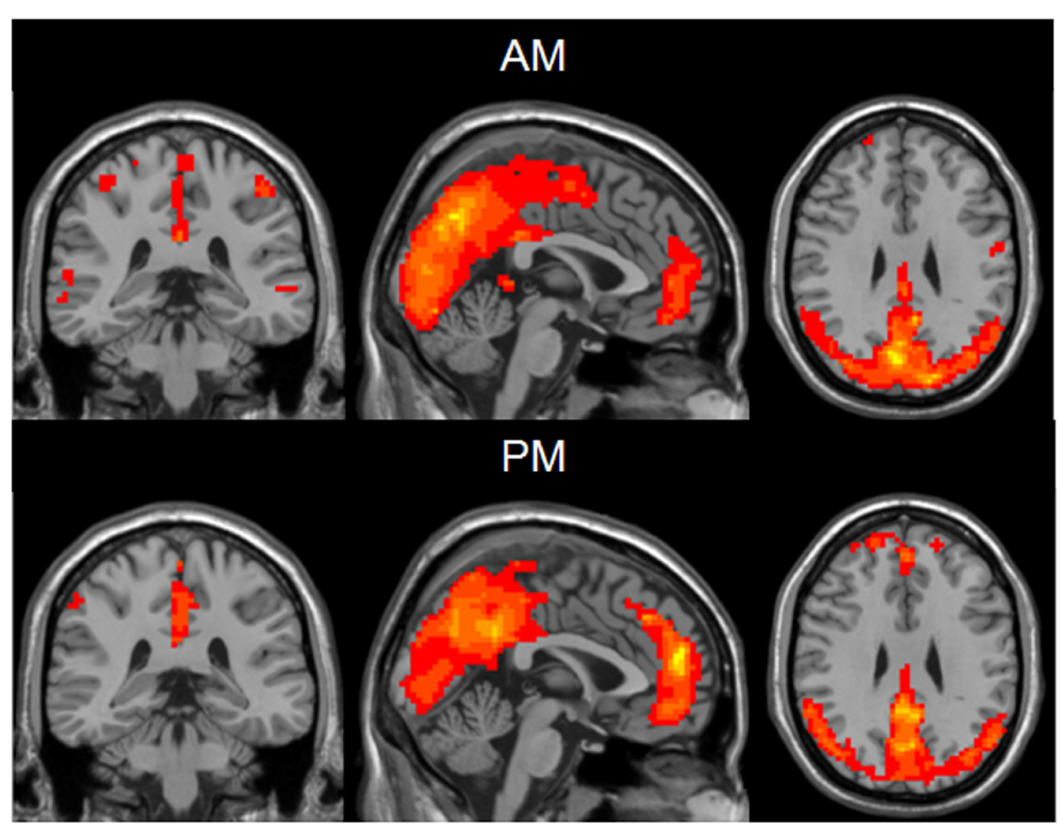

FIGURE 1 | One sample $t$-test results of mean ReHo maps within the AM group (Top) and PM group (Bottom) (MNI coordinates $x=-1, y=-35$, $\boldsymbol{z}=\mathbf{2 9}$ ). Statistical maps were overlapped on the anatomical template for visualization.

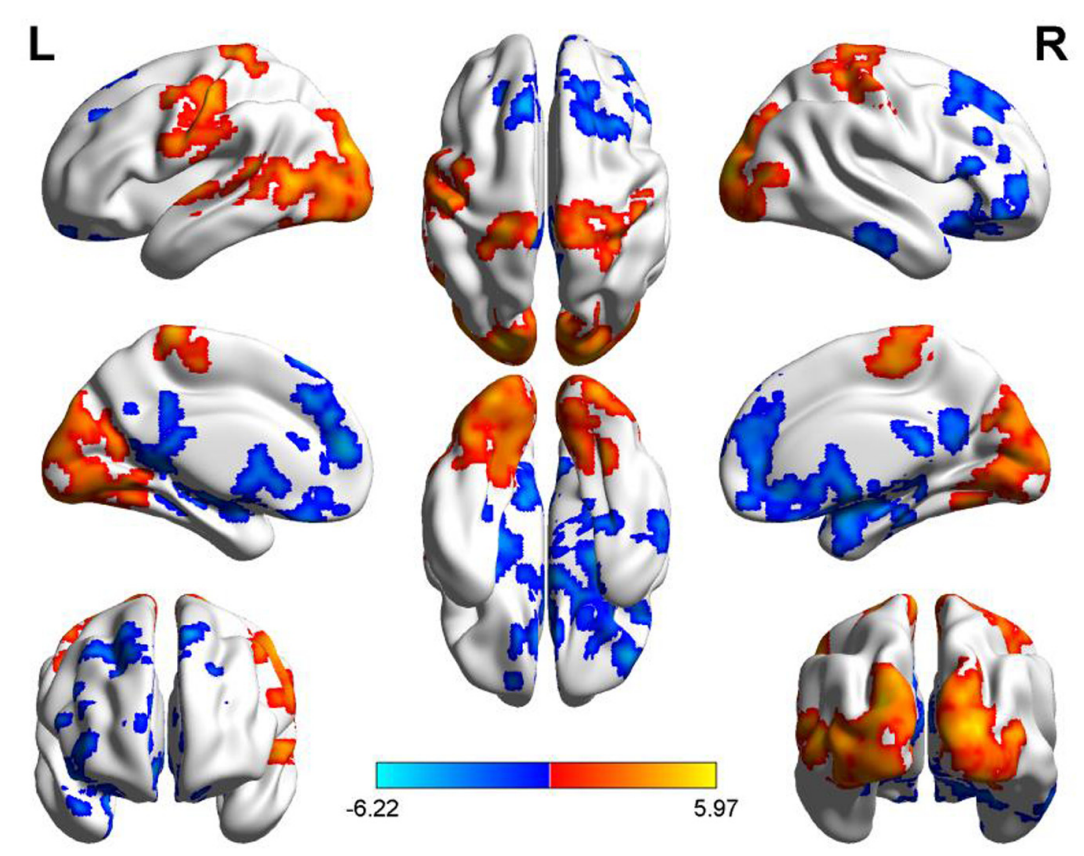

FIGURE 2 | Diurnal changes of ReHo. The final statistical maps are visualized by eight views (The first row from left to right is lateral view of left hemisphere, top side, lateral view of right hemisphere. The second row from left to right is medial view of left hemisphere, bottom side, medial view of right hemisphere. The third row is frontal and back side). Color bar indicates $t$-value (Red-yellow, AM > PM, blue-green, AM < PM).

interacting brain areas that are functionally connected. It can be divided into sub-networks contributing to specific tasks or intrinsic cognitive demand (Mayer et al., 2010; van Buuren et al., 2010). The current study demonstrated that overall the DMN is involved in greater neural activity at resting state in both morning and the evening as compared to the other brain areas, which is consistent with previous findings (Raichle et al., 2001; Fransson, 2005). But some of the structures were found to be more subject 
TABLE 1 | Summary of brain regions with significant increased ReHo in AM group compared to PM group.

\begin{tabular}{|c|c|c|c|}
\hline Brain regions & Peak coordinate & Cluster size & $\begin{array}{c}\text { Maximum } \\
t \text {-value }\end{array}$ \\
\hline Cuneus_R & $(21-9612)$ & 187 & 5.9733 \\
\hline Occipital_Mid_R & & 116 & \\
\hline Lingual_R & & 172 & \\
\hline Occipital_Sup_R & & 190 & \\
\hline Occipital_Inf_R & & 54 & \\
\hline Fusiform_R & & 50 & \\
\hline Calcarine_R & & 140 & \\
\hline Postcentral_R & & 297 & \\
\hline Precentral_R & & 71 & \\
\hline Paracentral_Lobule_R & & 78 & \\
\hline Cuneus_L & & 167 & \\
\hline Occipital_Mid_L & & 336 & \\
\hline Lingual_L & & 325 & \\
\hline Occipital_Sup_L & & 228 & \\
\hline Occipital_Inf_L & & 142 & \\
\hline Fusiform_L & & 117 & \\
\hline Calcarine_L & & 189 & \\
\hline Postcentral_L & & 203 & \\
\hline Precentral_L & & 92 & \\
\hline Paracentral_Lobule_L & $(-6-3672)$ & 82 & 4.636 \\
\hline Temporal_Mid_L & & 262 & \\
\hline Temporal_Sup_L & $(-54-60)$ & 85 & 4.3854 \\
\hline
\end{tabular}

to the diurnal alterations at the neural activity level, such as MPFC, medial temporal lobe, PCu and PCC. The divergence of the diurnal variation in these structures may suggest the functional coordination of the anatomically independent gyri. It has been reported that perturbations in DMN activity during wakefulness display co-occurring abnormalities of sleep in a number of disorders (Cherkassky et al., 2006; Garrity et al., 2007; Zhao et al., 2007). The DMN functional connectivity was found to be reduced in sleep deprivation (De Havas et al., 2012). The
TABLE 2 | Summary of brain regions with significant decreased ReHo in AM group compared to PM group.

\begin{tabular}{|c|c|c|c|}
\hline Brain regions & Peak coordinate & Cluster size & $\underset{t \text {-value }}{\text { Maximum }}$ \\
\hline Frontal_Sup_Orb_R & $(1821-15)$ & 27 & -5.887 \\
\hline Frontal_Mid_Orb_R & & 31 & \\
\hline Frontal_Sup_R & & 128 & \\
\hline Frontal_Mid_R & & 117 & \\
\hline Frontal_Sup_Medial_R & & 111 & \\
\hline Frontal_Inf_Orb_R & & 96 & \\
\hline ParaHippocampal_R & & 111 & \\
\hline Hippocampus_R & & 96 & \\
\hline Amygdala_R & & 36 & \\
\hline Precuneus_R & $(-21-3927)$ & 100 & -6.2167 \\
\hline Cingulum_Ant_R & & 102 & \\
\hline Cingulum_Post_R & & 32 & \\
\hline Caudate_R & & 14 & \\
\hline Frontal_Sup_Medial_L & & 234 & \\
\hline Frontal_Sup_L & & 65 & \\
\hline Frontal_Sup_Orb_L & & 17 & \\
\hline Frontal_Med_Orb_L & & 20 & \\
\hline Frontal_Mid_L & & 15 & \\
\hline ParaHippocampal_L & & 32 & \\
\hline Hippocampus_L & & 49 & \\
\hline Amygdala_L & $(-27-3-15)$ & 26 & -4.173 \\
\hline Precuneus_L & & 60 & \\
\hline Cingulum_Ant_L & & 41 & \\
\hline Cingulum_Post_L & & 28 & \\
\hline Caudate_L & & 64 & \\
\hline
\end{tabular}

$\mathrm{PCu}$ and PCC, which demonstrated increased activity in the PM group, play a pivotal role in the mediation of intrinsic activity through DMN (Fransson and Marrelec, 2008). In the contrast, the activity of medial temporal lobe decreased in the PM group. Considered in the context of these findings, it's reasonable to hypothesize that diurnal changes in DMN activity reflect
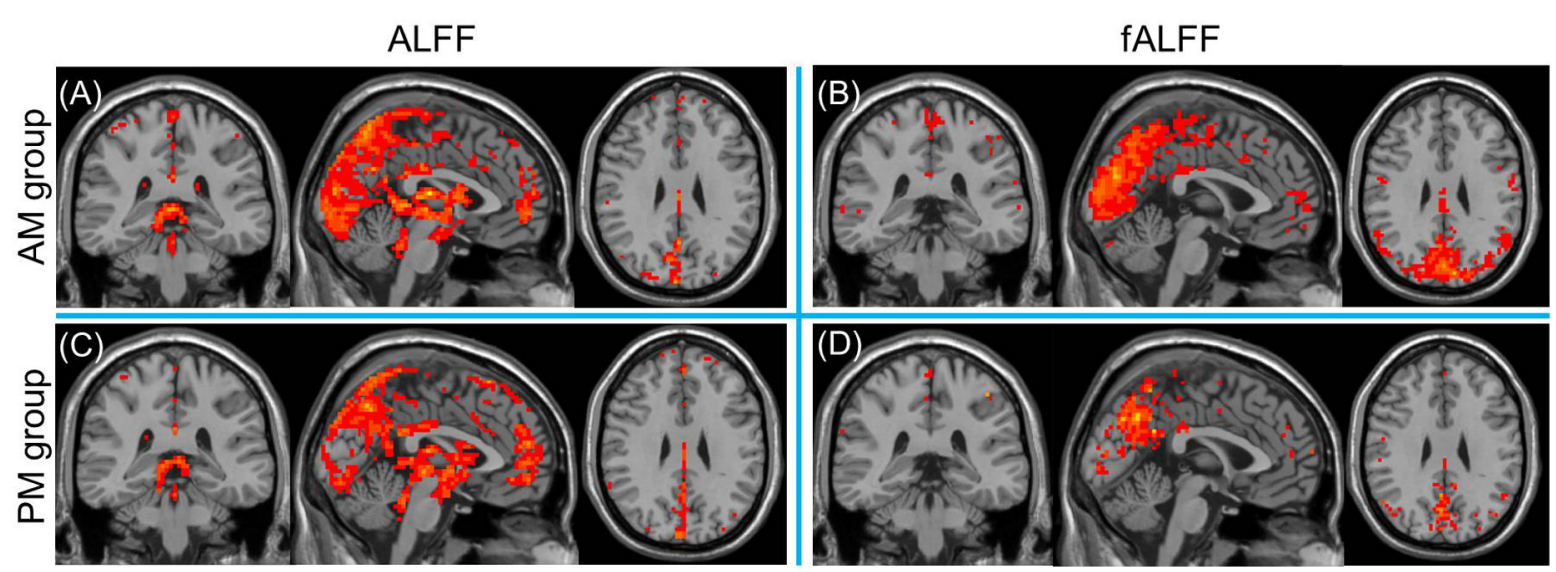

FIGURE 3 | One sample $\boldsymbol{t}$-test results of mean ALFF (A,C, left column) and fALFF (B,D, right column) maps within the AM group (top row) and PM group (bottom row) (MNI coordinates $x=-1, y=-35, z=29$ ). Statistical maps were overlapped on the anatomical template for visualization. 


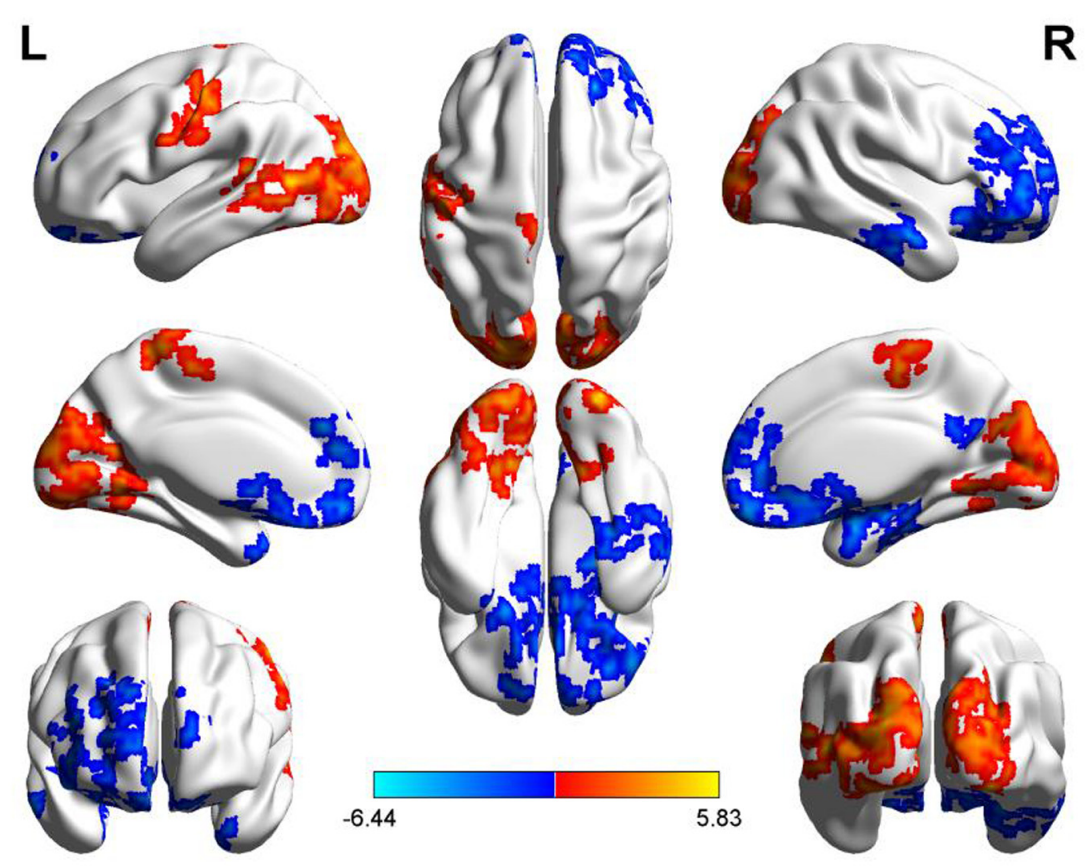

FIGURE 4 | Diurnal changes of ALFF. The final statistical maps are visualized by eight views (The first row from left to right is lateral view of left hemisphere, top side, lateral view of right hemisphere. The second row from left to right is medial view of left hemisphere, bottom side, medial view of right hemisphere. The third row is frontal and back side). Color bar indicates $t$-value (Red-yellow, AM > PM, blue-green, AM < PM).

TABLE 3 | Summary of brain regions with significant increased ALFF in AM group compared to PM group.

\begin{tabular}{lccc}
\hline Brain regions & Peak coordinate & Cluster size & $\begin{array}{c}\text { Maximum } \\
\boldsymbol{t} \text {-value }\end{array}$ \\
\hline Lingual_L & $(-18-75-12)$ & 160 & 5.8333 \\
Occipital_Mid_L & & 250 & \\
Occipital_Sup_L & & 131 & \\
Occipital_Inf_L & & 50 & \\
Calcarine_L & & 162 & \\
Cuneus_L & & 123 & \\
Temporal_Mid_L & 153 & \\
Postcentral_L & $(-51-1851)$ & 89 & 4.5896 \\
Precentral_L & & 23 & \\
Paracentral_Lobule_L & & 47 & \\
Occipital_Sup_R & & 74 & \\
Occipital_Mid_R & & 68 & \\
Occipital_Inf_R & & 17 & \\
Cuneus_R & & 134 & \\
Lingual_R & & 66 & \\
Paracentral_Lobule_R & $(6-2766)$ & 16 & 3.977 \\
\hline
\end{tabular}

adaptation or compensation response under continued wakeful condition. It may also imply that there is a balance of neural activity within DMN subregions, or a decoupling of regulation between these regions.

The occipital lobe shows significant decrease in ReHo and ALFF map in the PM group than that in the AM group. These findings are contrary to our initial assumption. The occipital lobe
TABLE 4 | Summary of brain regions with significant decreased ALFF in AM group compared to PM group.

\begin{tabular}{|c|c|c|c|}
\hline Brain regions & Peak coordinate & Cluster size & $\underset{t \text {-value }}{\text { Maximum }}$ \\
\hline Amygdala_R & $(240-21)$ & 24 & -6.4419 \\
\hline ParaHippocampal_R & & 64 & \\
\hline Hippocampus_R & & 56 & \\
\hline Fusiform_R & & 30 & \\
\hline Precuneus_R & & 26 & \\
\hline Frontal_Sup_R & & 94 & \\
\hline Frontal_Mid_R & & 142 & \\
\hline Frontal_Inf_Orb_R & & 86 & \\
\hline Frontal_Sup_Medial_R & & 87 & \\
\hline Rectus_R & (3 $54-15)$ & 77 & -6.2089 \\
\hline Cingulum_Ant_R & & 36 & \\
\hline Cingulum_Post_R & & 20 & \\
\hline Temporal_Inf_R & $(51-33-21)$ & 44 & -4.3871 \\
\hline Temporal_Mid_R & & 28 & \\
\hline Frontal_Sup_L & & 33 & \\
\hline Frontal_Med_Orb_L & & 31 & \\
\hline Frontal_Inf_Orb_L & & 26 & \\
\hline Cingulum_Ant_L & & 20 & \\
\hline Rectus_L & & 72 & \\
\hline
\end{tabular}

is mainly related to the visual information processing. The photic stimulus duration has an effect of "temporal integration" on neurons (Dkhissi-Benyahya et al., 2000), which means the neural activity should increase in the PM group. Also, decreased ADC 


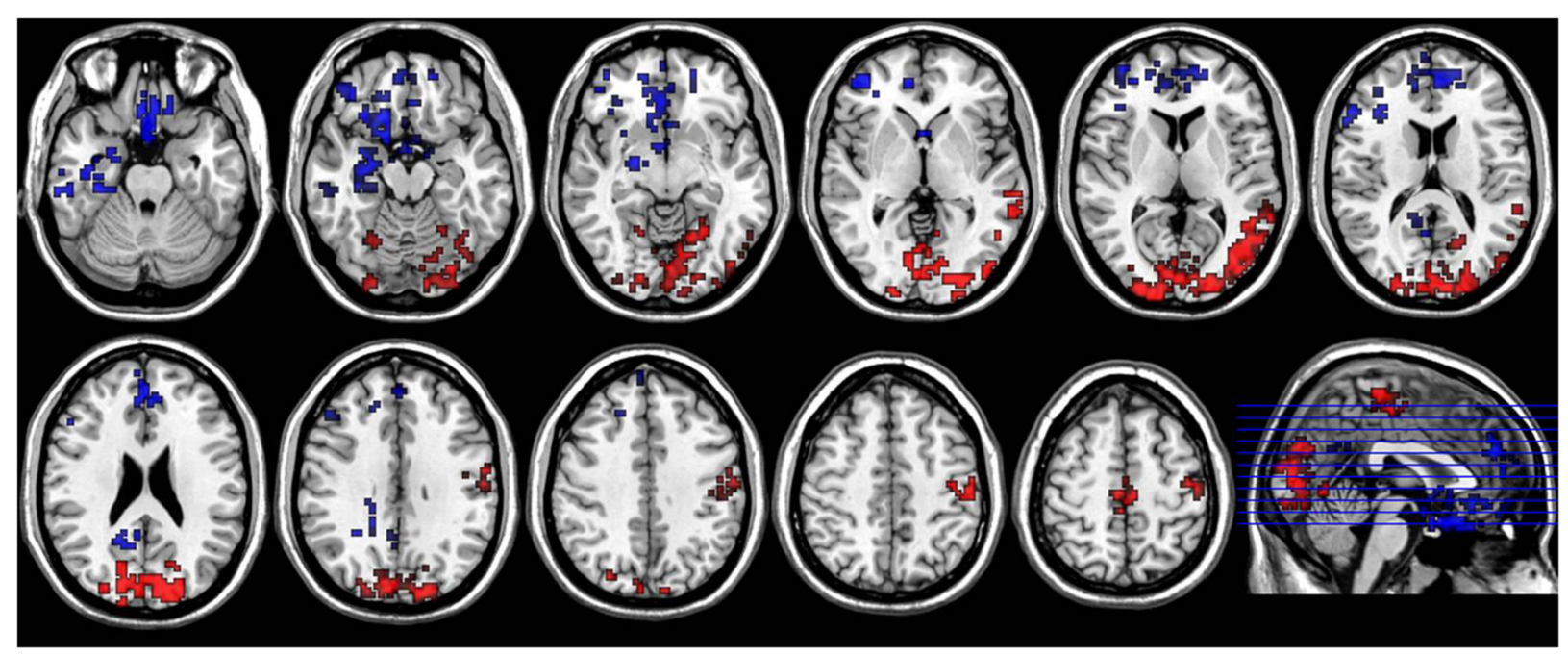

FIGURE 5 | Axial sections show areas with significant diurnal variations of both ReHo and ALFF. The left hand side of the image is the right side of the brain (radiology convention). The hot color indicates increased ReHo and ALFF in AM compared to PM, and cool color indicates decreased ReHo and ALFF.

(apparent diffusion coefficient) mainly occurred in the occipital lobe in the PM group in our previous study on diurnal variation of white matter using diffusion tensor imaging (DTI), which is believed to associate with the increased neuron activation (Jiang et al., 2014). But Buysse Daniel et al. (2004) measured relative glucose metabolism in the morning and in the evening with PET, and found significantly lower glucose metabolism in the evening than in the morning in occipital lobe, including cuneus, medial occipital gyrus, lingual gyrus, and occipitotemporal gyrus, which was interpreted to reflect increasing homeostatic sleep drive and/or the restorative effect of sleep. Muto et al. (2016) reported that the neural response of occipital gyrus are modulated by the interaction of circadian rhythmicity and sleep debt. These investigations suggest that sleep debt may have dominant control in the occipital lobe in the evening under current experiment condition.

Precentral and postcentral gyri have higher homogeneous neural activity and low frequency fluctuation in the morning than in the evening. This may suggest that during the evening hours, the somatosensory and motor functions represent a weakened response to the afferent and/or efferent signals. The exact biological mechanisms behind ReHo and ALFF remain unclear up to date. ReHo was defined as the temporal homogeneity of the regional BOLD signal (Zang et al., 2004), while ALFF reflects the amplitude of low-frequency fluctuation in the range of $0.01-0.08 \mathrm{~Hz}$ (Yu-Feng et al., 2007). Logothetis and Wandell (2004) reviewed that the hemodynamic response primarily reflects the neuronal input to the relevant area of the brain and synaptic potentials are the strongest cause of the BOLD response. The lower ReHo and ALFF in precentral and postcentral gyri found in this study may indicate that the synchronization of neural activity in these areas is disrupted and the amplitude of low-frequency fluctuation of BOLD signal decreases, possibly corresponding to the deteriorated somatosensory and motor response to the external stimulus and weakened local field potential as a result of increased level of exhaustiveness, sleepiness, and absentmindedness after a day experience.

The prefrontal cortex (including medial- and inferior-orbital frontal gyrus, and anterior cingulate) shows greater cerebral BOLD response in the PM group than in the AM group. These regions are reported to be involved in alertness, attention and higher-order cognition processes. However, Thomas et al. (2000) found that brain activity and function decrease in prefrontal cortex and thalamus after $24 \mathrm{~h}$ of sleep deprivation using positron emission tomography (PET), which implies that the need for recuperation in these areas is greater than other brain regions. On the contrary, Drummond and Brown (2001) reported that prefrontal cortex and parietal lobes showed increased activation in verbal learning and divided attention tasks after total sleep deprivation using fMRI, which may suggest a specific compensatory mechanism designed to combat increased sleepiness. Increased evening activity in the prefrontal cortex was also identified in this study. We hypothesize that the increased sleepiness or fatigue after a day of activity may induce neurochemical consequences for the brain to adapt to fulfillment the demand for the alertness as well as the higher cognitive performance as dark cycle approaches.

\section{LIMITATIONS OF CURRENT STUDY}

It should be noted that there are several limitations in this study. For example, the resting state fMRI signal is partially affected by cardiac pulsation, respiration, vasoconstriction, instrumental and thermal sources of noise. We didn't record the physiological parameters mentioned above during the fMRI data acquisition. During the data preprocessing procedure, a temporal band-pass filter was used to reduce low frequency drift and high frequency 
physiological noise. This may alleviate, although not completely eliminate the effect on accuracy and reliability for the current study.

Sex related difference in cerebral structure and function was not taken into account in this study, which may confound the interpretation of the results. Evidences have been accumulating that sex disparity matters in the circadian modulation of mammal brain (Mong et al., 2011). Women are found to have shorter intrinsic periods and earlier phase of melatonin rhythms as well as greater night-time impairment in cognitive performance as compared to men (Duffy et al., 2011; Santhi et al., 2016), but the intrinsic circadian periods of both gender are close to $24 \mathrm{~h}$. In addition, healthy people have different preferences in sleep pattern such as morning "lark" or evening "owl" chronotypes. This may also embed complicated mechanisms of rhythmic dynamics of human brain that this study did not reach.

Lastly, the fMRI data were acquired from only 16 young subjects at two time points. The sample size was relatively small. This can't and limit us in providing more significant findings. A larger sample size covering multiple age groups and time points are expected to provide stronger statistical power with reduced individual effects and enable a more detailed tracking of neural activities during the time course of a day-night cycle.

Further study is needed to optimize the experiment design, like recruitment of single gender subjects, melatonin assays to assess circadian phase, performing different cognitive tasks

\section{REFERENCES}

Buysse Daniel, J., Nofzinger, E. A., Germain, A., Meltzer, C. C., Wood, A., Ombao, H., et al. (2004). Regional brain glucose metabolism during morning and evening wakefulness in humans: preliminary findings. Sleep 27, 1245-1254.

Cherkassky, V. L., Kana, R. K., Keller, T. A., and Just, M. A. (2006). Functional connectivity in a baseline resting-state network in autism. Neuroreport 17, 1687-1690. doi: 10.1097/01.wnr.0000239956.45448.4c

Cirelli, C. (2009). The genetic and molecular regulation of sleep: from fruit flies to humans. Nat. Rev. Neurosci. 10, 549-560. doi: 10.1038/nrn2683

De Havas, J. A., Parimal, S., Soon, C. S., and Chee, M. W. L. (2012). Sleep deprivation reduces default mode network connectivity and anti-correlation during rest and task performance. Neuroimage 59, 1745-1751. doi: 10.1016/j. neuroimage.2011.08.026

Dkhissi-Benyahya, O., Sicard, B., and Cooper, H. M. (2000). Effects of irradiance and stimulus duration on early gene expression (Fos) in the suprachiasmatic nucleus: temporal summation and reciprocity. J. Neurosci. 20, 7790-7797.

Drummond, S. P. A., and Brown, G. G. (2001). The effects of total sleep deprivation on cerebral responses to cognitive performance. Neuropsychopharmacology 25, S68-S73. doi: 10.1016/S0893-133X(01)00325-6

Duffy, J. F., Cain, S. W., Chang, A.-M., Phillips, A. J., Münch, M. Y., Gronfier, C., et al. (2011). Sex difference in the near-24-hour intrinsic period of the human circadian timing system. Proc. Natl. Acad. Sci. U.S.A. 108(Suppl. 3), 1560215608. doi: $10.1073 /$ pnas. 1010666108

Fransson, P. (2005). Spontaneous low-frequency BOLD signal fluctuations: an fMRI investigation of the resting-state default mode of brain function hypothesis. Hum. Brain Mapp. 26, 15-29. doi: 10.1002/hbm.20113

Fransson, P., and Marrelec, G. (2008). The precuneus/posterior cingulate cortex plays a pivotal role in the default mode network: evidence from a partial correlation network analysis. Neuroimage 42, 1178-1184. doi: 10.1016/j. neuroimage.2008.05.059 that are differentially affected by sleep drive and circadian rhythmicity.

\section{CONCLUSION}

Neural activity of healthy young human brain exhibits a diurnal pattern which may be related to circadian plasticity or accumulated waking, which may substrate the neurobiological and behavioral mechanisms of sleep hygiene and sleep disorders. Factors of time of day should be taken into account in the functional neuroimaging based neural studies even under resting conditions.

\section{AUTHOR CONTRIBUTIONS}

Conceived and designed the experiments: LZ and CJ. Performed the experiments: CJ, LY, and LZ. Analyzed the data: CJ, LY, SS, CS, XL, GX, and LZ. Wrote the paper: CJ and LZ.

\section{FUNDING}

This study was partially supported by National Natural Science Foundation of China (Grant number 81371359), National Basic Research Program of China (Grant number 2015CB755500) and the Basic Research Program of Shenzhen (Grant number JCYJ20160429191938883).

Garrity, A. G., Pearlson, G. D., McKiernan, K., Lloyd, D., Kiehl, K. A., and Calhoun, V. D. (2007). Aberrant "default mode" functional connectivity in schizophrenia. Am. J. Psychiatry 164, 450-457. doi: 10.1176/ajp.2007.164.3.450

Hastings, M. H., Reddy, A. B., and Maywood, E. S. (2003). A clockwork web: circadian timing in brain and periphery, in health and disease. Nat. Rev. Neurosci. 4, 649-661. doi: 10.1038/nrn1177

He, Y., Wang, L., Zang, Y., Tian, L., Zhang, X., Li, K., et al. (2007). Regional coherence changes in the early stages of Alzheimer's disease: a combined structural and resting-state functional MRI study. Neuroimage 35, 488-500. doi: 10.1016/j.neuroimage.2006.11.042

Hofman, M. A., and Swaab, D. F. (1993). Diurnal and seasonal rhythms of neuronal activity in the suprachiasmatic nucleus of humans. J. Biol. Rhythms 8, 283-295. doi: 10.1177/074873049300800402

Jiang, C., Zhang, L., Zou, C., Long, X., Liu, X., Zheng, H., et al. (2014). Diurnal microstructural variations in healthy adult brain revealed by diffusion tensor imaging. PLoS ONE 9:e84822. doi: 10.1371/journal.pone.0084822

Li, J. Z., Bunney, B. G., Meng, F., Hagenauer, M. H., Walsh, D. M., Vawter, M. P., et al. (2013). Circadian patterns of gene expression in the human brain and disruption in major depressive disorder. Proc. Natl. Acad. Sci. U.S.A. 110, 9950-9955. doi: 10.1073/pnas.1305814110

Li, R., Ma, Z., Yu, J., He, Y., and Li, J. (2014). Altered local activity and functional connectivity of the anterior cingulate cortex in elderly individuals with subthreshold depression. Psychiatry Res. 222, 29-36. doi: 10.1016/j.pscychresns. 2014.02.013

Logothetis, N. K., Pauls, J., Augath, M., Trinath, T., and Oeltermann, A. (2001). Neurophysiological investigation of the basis of the fMRI signal. Nature 412, 150-157. doi: 10.1038/35084005

Logothetis, N. K., and Wandell, B. A. (2004). Interpreting the BOLD Signal. Annu. Rev. Physiol. 66, 735-769. doi: 10.1146/annurev.physiol.66.082602.092845

Marek, T., Fafrowicz, M., Golonka, K., Mojsa-Kaja, J., Oginska, H., Tucholska, K., et al. (2010). Diurnal patterns of activity of the orienting and executive attention neuronal networks in subjects performing a Stroop-like task: a functional 
magnetic resonance imaging study. Chronobiol. Int. 27, 945-958. doi: 10.3109/ 07420528.2010.489400

Mayer, J. S., Roebroeck, A., Maurer, K., and Linden, D. E. J. (2010). Specialization in the default mode: task-induced brain deactivations dissociate between visual working memory and attention. Hum. Brain Mapp. 31, 126-139. doi: 10.1002/ hbm. 20850

Mohawk, J. A., Green, C. B., and Takahashi, J. S. (2012). Central and peripheral circadian clocks in mammals. Annu. Rev. Neurosci. 35, 445-462. doi: 10.1146/ annurev-neuro-060909-153128

Mong, J. A., Baker, F. C., Mahoney, M. M., Paul, K. N., Schwartz, M. D., Semba, K., et al. (2011). Sleep, rhythms, and the endocrine brain: influence of sex and gonadal hormones. J. Neurosci. 31, 16107-16116. doi: 10.1523/JNEUROSCI. 4175-11.2011

Muto, V., Jaspar, M., Meyer, C., Kussé, C., Chellappa, S. L., Degueldre, C., et al. (2016). Local modulation of human brain responses by circadian rhythmicity and sleep debt. Science 353, 687-690. doi: 10.1126/science.aad2993

Park, B., Kim, J. I., Lee, D., Jeong, S.-O., Lee, J. D., and Park, H.-J. (2012). Are brain networks stable during a 24-hour period? Neuroimage 59, 456-466. doi: 10.1016/j.neuroimage.2011.07.049

Raichle, M. E., MacLeod, A. M., Snyder, A. Z., Powers, W. J., Gusnard, D. A., and Shulman, G. L. (2001). A default mode of brain function. Proc. Natl. Acad. Sci. U.S.A. 98, 676-682. doi: 10.1073/pnas.98.2.676

Santhi, N., Lazar, A. S., McCabe, P. J., Lo, J. C., Groeger, J. A., and Dijk, D.-J. (2016). Sex differences in the circadian regulation of sleep and waking cognition in humans. Proc. Natl. Acad. Sci. U.S.A. 113, E2730-E2739. doi: 10.1073/pnas. 1521637113

Saper, C. B., Scammell, T. E., and Lu, J. (2005). Hypothalamic regulation of sleep and circadian rhythms. Nature 437, 1257-1263. doi: 10.1038/nature 04284

Smale, L., Nunez, A. A., and Schwartz, M. D. (2008). Rhythms in a diurnal brain. Biol. Rhythm Res. 39, 305-318. doi: 10.1080/0929101070168 2666

Thomas, M., Sing, H., Belenky, G., Holcomb, H., Mayberg, H., Dannals, R., et al. (2000). Neural basis of alertness and cognitive performance impairments during sleepiness. I. Effects of $24 \mathrm{~h}$ of sleep deprivation on waking human regional brain activity. J. Sleep Res. 9, 335-352. van Buuren, M., Gladwin, T. E., Zandbelt, B. B., Kahn, R. S., and Vink, M. (2010). Reduced functional coupling in the default-mode network during selfreferential processing. Hum. Brain Mapp. 31, 1117-1127. doi: 10.1002/hbm. 20920

Vicentic, A., Lakatos, A., Hunter, R., Philpot, K., Dominguez, G., and Kuhar, M. J. (2005). CART peptide diurnal rhythm in brain and effect of fasting. Brain Res. 1032, 111-115. doi: 10.1016/j.brainres.2004.10.053

Yang, H., Long, X. Y., Yang, Y., Yan, H., Zhu, C. Z., Zhou, X. P., et al. (2007). Amplitude of low frequency fluctuation within visual areas revealed by restingstate functional MRI. Neuroimage 36, 144-152. doi: 10.1016/j.neuroimage.2007. 01.054

Yu-Feng, Z., Yong, H., Chao-Zhe, Z., Qing-Jiu, C., Man-Qiu, S., Meng, L., et al. (2007). Altered baseline brain activity in children with ADHD revealed by resting-state functional MRI. Brain Dev. 29, 83-91. doi: 10.1016/j.braindev. 2006.07.002

Zang, Y., Jiang, T., Lu, Y., He, Y., and Tian, L. (2004). Regional homogeneity approach to fMRI data analysis. Neuroimage 22, 394-400. doi: 10.1016/j. neuroimage.2003.12.030

Zhao, X.-H., Wang, P.-J., Li, C.-B., Hu, Z.-H., Xi, Q., Wu, W.-Y., et al. (2007). Altered default mode network activity in patient with anxiety disorders: an fMRI study. Eur. J. Radiol. 63, 373-378. doi: 10.1016/j.ejrad.2007.02.006

Zou, Q. H., Zhu, C. Z., Yang, Y., Zuo, X. N., Long, X. Y., Cao, Q. J., et al. (2008). An improved approach to detection of amplitude of low-frequency fluctuation (ALFF) for resting-state fMRI: fractional ALFF. J. Neurosci. Methods 172, 137-141. doi: 10.1016/j.jneumeth.2008.04.012

Conflict of Interest Statement: The authors declare that the research was conducted in the absence of any commercial or financial relationships that could be construed as a potential conflict of interest.

Copyright (C) 2016 Jiang, Yi, Su, Shi, Long, Xie and Zhang. This is an open-access article distributed under the terms of the Creative Commons Attribution License (CC BY). The use, distribution or reproduction in other forums is permitted, provided the original author(s) or licensor are credited and that the original publication in this journal is cited, in accordance with accepted academic practice. No use, distribution or reproduction is permitted which does not comply with these terms. 